\title{
Nasal Turbinate
}

National Cancer Institute

\section{Source}

National Cancer Institute. Nasal Turbinate. NCI Thesaurus. Code C49594.

One of three (Inferior, Middle, Superior) paired bony shelves located within the nasal cavity throug h which inhaled air is taken into the nasopharynx. It is lined with ciliated, pseudostratified columnar epithelium and functions to humidify, heat, and filter incoming air, as well as protecting the olfactory bulb and sinuses from incorrectly pressurized incoming air. 